\title{
Qualidade de vida na asma brônquica - a concordância das percepções das crianças, adolescentes e seus pais
}

\author{
Quality of life in asthmatics - concordance among children, adolescents, and their parents
}

\author{
Laura Janne L. Aragão¹, Maria Wanderleya L. Coriolano-Marinus², Gabriela Cunha S. Sette ${ }^{3}$, Maria Cristina F. Raposo4, Murilo Carlos
}

A. de Britto ${ }^{5}$, Luciane Soares de Lima $^{6}$

\section{RESUMO}

Objetivo: Avaliar a percepção da qualidade de vida, com relação à asma, pelas crianças, adolescentes e seus pais, verificando as concordâncias existentes em cada domínio do questionário Pediatric Asthma Quality of Life Questionnaire Adaptado (PAQLQ-A).

Métodos: Estudo transversal realizado em dois ambulatórios universitários de referência para seguimento de crianças e adolescentes asmáticos em Recife (PE). Após o cumprimento das exigências éticas, os dados sobre a qualidade de vida foram obtidos por aplicação do PAQLQ, em sua versão traduzida adaptada e validada para a língua portuguesa. O questionário foi respondido por 126 pacientes ( 70 crianças e 56 adolescentes) e seus respectivos pais. Um banco de dados criado no programa SPSS, versão 13.0, foi analisado com o teste de concordância Kappa.

Resultados: A concordância das respostas entre crianças e pais variou de ruim a sofrível, observando-se as piores concordâncias no domínio sintomas para o item "chiado no peito" e, no domínio emoções, para o item "sentiu-se irritado". As respostas dos adolescentes e seus pais tiveram a concordância avaliada entre ruim e regular; sendo a pior concordância observada no domínio atividades, no item "incomodado por não poder estar com os outros" e a melhor concordância notada no domínio emoções, no item "desconfortável”.

Instituição: Programa de Pós-graduação em Saúde da Criança e do Adolescente da Universidade Federal de Pernambuco (UFPE), Recife, PE, Brasil

'Mestre em Saúde da Criança e do Adolescente pela UFPE; Professora Assistente 2 do Departamento de Medicina da Universidade Estadual do Rio Grande do Norte (UERN), Mossoró, RN, Brasil

2Doutoranda em Saúde da Criança e do Adolescente pela UFPE; Professora Substituta do Departamento de Enfermagem da UFPE, Recife, PE, Brasil ${ }^{3}$ Doutoranda em Saúde da Criança e do Adolescente pela UFPE; Professora Assistente do Departamento de Enfermagem da UFPE, Recife, PE, Brasil ${ }^{4}$ Doutora em Economia pela UFPE; Professora Adjunta do Departamento de Estatística da UFPE, Recife, PE, Brasil

${ }^{5}$ Doutor em Saúde Pública pela Fundação Oswaldo Cruz (Fiocruz); Professor do Departamento de Pós-graduação em Saúde Materno-Infantil do Instituto de Medicina Integral (IMIP), Recife, PE, Brasil
Conclusões: O PALQ-A não foi bem compreendido pela população estudada e não houve concordância na percepção sobre qualidade de vida das crianças e adolescentes asmáticos e seus pais. Recomenda-se a elaboração de instrumentos adequados às especificidades regionais ou ainda a revalidação de instrumentos internacionais, de forma que atendam à diversidade regional.

Palavras-chave: qualidade de vida; criança; adolescente; asma; pais.

\section{ABSTRACT}

Objective: To evaluate the perception of the quality of life of asthmatic children and adolescents and their parents, investigating the agreement rates in each domain using the Pediatric Asthma Quality of Life Questionnaire - Adapted (PAQLQ-A).

Methods: Cross-sectional study conducted at two outpatient clinics of a university reference center for asthmatic children and adolescents in Recife, Brazil. After meeting the ethical requirements, health-related quality of life data were collected using the version of the PAQLQ adapted and translated into Portuguese. The questionnaire was answered by 126 patients ( 70 children and 56 adolescents) and their parents. Agreement was analyzed by the Kappa statistic test using the computer program SPSS, version 13.0, software.

'Doutora em Ciências Pneumológicas pela Universidade Federal de São Paulo (Unifesp); Professora Titular do Departamento de Enfermagem da UFPE, Recife, PE, Brasil

Endereço para correspondência:

Luciane Soares de Lima

Rua do Futuro, 77, apto 1.101 - Graças

CEP 52050-010 - Recife/PE

E-mail: luciane.lima@globo.com

Conflito de interesse: nada a declarar

Recebido em: 14/12/2010

Aprovado em: 15/7/2011 
Results: Agreement between children and parents' answers varied from "unsatisfactory" to "poor". The worst agreement rate was found in the domain symptoms, especially regarding the item "wheezing", and in the domain emotions, regarding the item "feel bothered". Agreement between adolescents and their parents' answers varied from "unsatisfactory" to "fair", with the worst agreement rate in the domain activities, especially in the item "feel bothered by not being able to be with other people", and the best agreement rate was found in the domain emotions, in the item "feel uncomfortable."

Conclusions: The PAQLQ-A was not well understood by the studied population and agreement regarding the perception of the quality of life of asthmatic children and adolescents between patients and their parents was poor. Appropriate instruments adapted to regional characteristics or even revalidation of international instruments considering regional diversity should be developed.

Key-words: quality of life; child; adolescent; asthma; parents.

\section{Introdução}

A asma é uma doença inflamatória crônica caracterizada por hiper-responsividade das vias aéreas inferiores e por limitação variável ao fluxo aéreo, reversível espontaneamente ou com tratamento, manifestando-se clinicamente por episódios recorrentes de sibilância, dispneia, aperto no peito e tosse, particularmente à noite e pela manhã, ao despertar ${ }^{(1)}$.

O tratamento, de acordo com a classificação da asma, deve abordar aspectos como a farmacoterapia antiinflamatória inalatória ou sistêmica, broncodilatadores $\beta_{2}$-agonistas de curta ou longa duração e o uso dos antileucotrienos, associados ao controle dos fatores desencadeantes domiciliares e ocupacionais. A imunoterapia é indicada na asma atópica, demonstrada por imunoglobulina da classe $\mathrm{E}$ ( $\mathrm{IgE}$ ) específica aumentada e testes cutâneos de hipersensibilidade imediata positivos $^{(1)}$.

Existem evidências que os parâmetros clínicos e laboratoriais têm fraca correlação com o que a criança está sentindo no seu dia a dia e com a percepção dos pais com relação à qualidade de vida (QV) de seus filhos. A QV varia entre as pessoas, de acordo com as suas expectativas em relação à doença e à vida, que podem mudar com o passar do tempo e conforme as experiências vivenciadas ${ }^{(2)}$. Em crianças, a medida da QV foi, por muito tempo, baseada na medida convencional da gravidade da asma, na mensuração da função pulmonar, na presença e na intensidade dos sintomas, na necessidade de medicação e de discussão com os pais ${ }^{(3)}$. Vale lembrar que informações sobre QV têm sido incluídas como indicadores de avaliação da eficácia, eficiência e impacto de determinados tratamentos para grupos de portadores de agravos diversos, quando são comparados procedimentos para o controle de problemas de saúde específicos ${ }^{(4)}$. Atualmente, é tema de pesquisa imprescindível, visto que seus resultados contribuem para aprovar e definir tratamentos, avaliar custos e benefícios do cuidado prestado ${ }^{(5,6)}$.

Juniper et al ${ }^{(7,8)}$ desenvolveram o Pediatric Asthma Quality of Life Questionnaire (PAQLQ), validado e publicado em 1996 na língua inglesa e, posteriormente, em 20 outros idiomas. O PAQLQ é composto de 23 questões divididas em três domínios: limitação das atividades físicas (cinco questões), sintomas (10 questões) e emoções (oito questões). No domínio atividades, três questões foram individualizadas, podendo o paciente escolher a atividade que mais o incomoda executar. As respostas são medidas por meio de uma escala de sete pontos, sendo "um" equivalente a prejuízo máximo e "sete", nenhum prejuízo. Para uso no Brasil, o questionário foi testado e adaptado, mantendo-se o formato original, mas com a retirada de cinco atividades que não fazem parte da cultura local (hóquei de bola, futebol americano, esqui, beisebol e patinação no gelo). Os demais itens foram mantidos de acordo com a tradução, mas ordenados de maneira a tornar a aplicação mais fácil. O novo questionário traduzido e adaptado foi denominado Pediatric Asthma Quality of Life Questionnaire - Adaptado (PAQLQ-A) e teve a mesma escala de pontuação (um a sete) ${ }^{(9)}$.

No que tange especificamente à $\mathrm{QV}$ da criança e adolescente asmáticos, há muitas especulações e pontos de vista variados. Por isso, somente resultados de pesquisas metodologicamente bem conduzidas, podem suplantar tais especulações e, talvez, oferecer sugestões para programas e políticas assistenciais pautados nos achados e na busca da integração dessas crianças com seus familiares. Essas considerações motivaram a realização deste estudo, objetivando avaliar a percepção da QV, com relação à asma, pelas crianças, adolescentes e seus pais, e verificando as concordâncias existentes em cada domínio quando aplicado o PAQLQ- ${ }^{(8)}$.

\section{Método}

Estudo transversal realizado no Ambulatório de Alergia e Imunologia em Pediatria do Hospital das Clínicas da Universidade Federal de Pernambuco (UFPE) e no Ambulatório 
de Pneumologia Pediátrica do Instituto de Medicina Integral Professor Fernando Figueira. Os hospitais são de utilidade pública, credenciados pelo Ministério da Educação, referência no ensino em Recife (PE) para tratamento das doenças alérgicas e pulmonares, respectivamente. O projeto foi aprovado pelo Comitê de Ética em Pesquisa com Seres Humanos da UFPE.

Foram incluídas crianças na faixa etária de 7 a 10 anos incompletos e adolescentes de 10 a 19 anos $^{(10)}$. De acordo com a classificação de gravidade da asma, foram incluídas crianças e adolescentes com asma brônquica intermitente, persistente leve, moderada e grave ${ }^{(1)}$. Foram excluídos aqueles com diagnóstico de doenças imunológicas e respiratórias crônicas como: bronquiolites, bronquiectasias e pneumonias intersticiais. Assim, a população do estudo foi composta por 70 crianças, 56 adolescentes e seus respectivos pais. As entrevistas foram realizadas em encontro único, no período de abril a agosto de 2006.

A coleta das informações foi realizada pelo pesquisador responsável e dois alunos treinados para a aplicação do PAQLQ-A traduzido e validado em 2004 por La Scala, Naspitz e Solé(9). O PAQLQ-A é composto de 23 questões divididas em três domínios: sintomas, emoções e atividades. Para cada pergunta foi apresentado um cartão resposta com pontuação de um a sete (um=máximo prejuízo e sete=nenhum prejuízo). As perguntas foram relacionadas à última semana, e divididas de acordo com a pergunta em cartão verde (quantificando a frequência) e azul (quantificando a intensidade dos sintomas).

A pesquisa foi explicada aos pais ou responsáveis, às crianças e aos adolescentes, detalhando-se objetivos, importância e operacionalização. Após concordância do responsável, crianças e adolescentes, foi assinado o termo de consentimento livre e esclarecido por ambos. Crianças e adolescentes incluídos foram submetidos à anamnese e exame clínico e avaliados quanto à classificação da gravidade da asma ${ }^{(1)}$. Em seguida, foram convidados a responder ao PAQLQ-A, sendo as perguntas feitas pelo entrevistador. A seguir, o mesmo PAQLQ-A foi aplicado aos pais pelo entrevistador, sem a presença da criança/adolescente, visando à análise comparativa entre as respostas das crianças/ adolescentes e seus pais.

Para determinar o tamanho da amostra para cada grupo de idade, utilizou-se um procedimento do tipo sequencial, iniciando-se com uma amostra piloto de 20 crianças e 20 adolescentes. Em seguida, sorteou-se uma nova amostra de mesmo tamanho, realizando testes de diferenças de médias, ou proporções, conforme a variável de interesse, repetindo-se este procedimento até a estabilização das medidas, o que ocorreu com uma amostra de 70 crianças e 56 adolescentes.

Os dados foram processados no software SPSS versão 13.0 e, para medir o grau de concordância, foi utilizado o coeficiente de concordância Kappa. Este índice é um indicador de concordância ajustada (variação: $-1 \mathrm{a}+1$ ) e desconta o fator chance. Conforme sugerido por Andrade e Zicker ${ }^{(11)}$, os valores podem variar de $<0,00$ (ruim ou não ocorrem concordâncias); 0,00 a 0,20 (fraca); 0,21 a 0,40 (sofrível); 0,41 a 0,60 (regular); 0,61 a 0,80 (boa); 0,81 a 0,99 (ótima) e 1,00 (concordância perfeita). Nos casos em que os dados se apresentaram de forma não paramétrica, adotou-se o coeficiente de correlação por postos de Spearman. Em todos os casos, foi adotado o nível de 95\% de confiança.

\section{Resultados}

A amostra estudada constituiu-se de 126 crianças e adolescentes e seus pais (126). A mediana de idade das crianças foi de 9 anos e dos adolescentes, 13 . Entre as crianças, houve predominância da asma persistente leve $(25 / 36 \%)$ e, entre os adolescentes, a intermitente (22/39\%). A rinite alérgica foi a doença associada mais comum encontrada nos pacientes -64 crianças $(91 \%)$ e 49 adolescentes (87\%). As características demográficas são evidenciadas na Tabela 1 .

Na Tabela 2, observa-se a frequência de perguntas respondidas pelas crianças, adolescentes e seus pais para os diversos domínios do instrumento avaliado. Em alguns itens do questionário, a amostra ficou reduzida de forma importante, pois ambos (pais e crianças) não compreenderam a pergunta. Destaca-se, por exemplo, que em $43 \%$ da amostra de crianças não se obteve a informação combinada com seus pais na variável "emoções por sentir-se de fora”. Observou-se, ainda, no domínio emoções, a falta de entendimento dos termos "desconfortável" e "sentir-se frustrado", o que ocasionou a exclusão de 15 e 13 pares de crianças/pais e adolescentes/pais, respectivamente, da análise de concordância para as respostas nestes itens do questionário.

A Tabela 3 indica que a concordância entre as respostas das crianças e de seus pais para os domínios de sintomas, emoções e atividades do questionário PAQLQ-A variou de ruim a sofrível. Quanto aos itens com pior concordância, constatou-se que, no domínio sintomas, o item foi o "chiado no peito"; no domínio emoções, foi a menção "por sentir-se 
Tabela 1 - Distribuição de variáveis demográficas e clínicas da amostra de crianças e adolescentes com asma

\begin{tabular}{|c|c|c|c|c|}
\hline & \multicolumn{2}{|c|}{ Crianças } & \multicolumn{2}{|c|}{ Adolescentes } \\
\hline & $n=70$ & $\%$ & $n=56$ & $\%$ \\
\hline \multicolumn{5}{|l|}{ Sexo } \\
\hline Masculino & 40 & 57,1 & 32 & 57,1 \\
\hline Feminino & 30 & 42,9 & 24 & 42,9 \\
\hline \multicolumn{5}{|c|}{ Classificação de gravidade asma } \\
\hline Intermitente & 15 & 21,4 & 22 & 39,3 \\
\hline Persistente leve & 25 & 35,7 & 12 & 21,4 \\
\hline Persistente moderada & 22 & 31,4 & 18 & 32,1 \\
\hline Persistente grave & 8 & 11,4 & 4 & 7,1 \\
\hline \multicolumn{5}{|l|}{ Doenças associadas } \\
\hline Rinite alérgica & 64 & 91,4 & 49 & 87,5 \\
\hline Dermatite atópica & 9 & 12,9 & 6 & 10,7 \\
\hline Conjuntivite & 10 & 14,3 & - & - \\
\hline Alergia alimentar & 10 & 14,3 & 3 & 5,4 \\
\hline
\end{tabular}

Tabela 2 - Distribuição da frequência de respostas das crianças e adolescentes com asma e seus pais, segundo os três domínios do Pediatric Asthma Quality of Life Questionnaire Adaptado

\begin{tabular}{lcccc}
\hline & \multicolumn{2}{c}{$\begin{array}{c}\text { Informações combinadas } \\
\text { Crianças e pais (n=70) }\end{array}$} & $\begin{array}{c}\text { Informações combinadas } \\
\text { Adolescentes e pais (n=56) }\end{array}$ \\
\cline { 2 - 5 } & $\mathbf{n}$ & Sem resposta (\%) & $\mathbf{n}$ & Sem resposta (\%) \\
\hline Domínio dos sintomas & & & & \\
1. Tosse & 70 & 0 & 56 & 0 \\
2. Crise de asma & 69 & $1,0(1,4)$ & 56 & 0 \\
3. Chiado no peito & 70 & 0 & 56 & 0 \\
4. Aperto no peito & 65 & $5,0(7,1)$ & 54 & $2,0(3,6)$ \\
5. Sintomas de respiração curta & 70 & 0 & 56 & 0 \\
6. Sentiu-se cansado & 70 & 0 & 55 & $1,0(1,8)$ \\
7. Acordou a noite por asma & 70 & 0 & 56 & 0 \\
8. Sentiu-se sem respiração & 69 & $1,0(1,4)$ & 56 & 0 \\
9. Problemas para dormir & 70 & 0 & 56 & 0 \\
10. Dificuldades em respirar profundamente & 70 & 0 & 56 & 0 \\
Domínio das emoções & & & & \\
1. Sentir-se frustrado & 57 & $13,0(18,6)$ & 55 & $1,0(1,8)$ \\
2. Sentiu-se preocupado & 69 & $1,0(1,4)$ & 56 & 0 \\
3. Sentiu-se zangado & 70 & 0 & 56 & 0 \\
4. Emoções por sentir-se de fora & 40 & $30,0(42,9)$ & 47 & $9,0(16,1)$ \\
5. Frustrado por não poder estar com os outros & 57 & $13,0(18,6)$ & 55 & $1,0(1,8)$ \\
6. Desconfortável & 55 & $15,0(21,4)$ & 56 & 0 \\
7. Apavorado por crise de asma & 69 & $1,0(1,4)$ & 56 & 0 \\
8. Sentiu-se irritado & 69 & $1,0(1,4)$ & 56 & 0 \\
Domínio das Atividades & & & & $2,0(3,6)$ \\
1. Incomodado por não poder estar com os outros & 66 & $4,0(5,7)$ & 54 & $2,0(3,6)$ \\
2. Incomodado ao realizar as atividades da ultima & 66 & $4,0(5,7)$ & 54 & \\
semana & & & & \\
\hline
\end{tabular}


Tabela 3 - Concordância na aplicação do questionário PediatricAsthma Quality of Life Questionnaire Adaptado entre as respostas obtidas de crianças e seus pais

\begin{tabular}{lccc}
\hline & Coeficiente Kappa & Concordância & Valor $\boldsymbol{p}$ \\
\hline Domínio dos sintomas & & & $<0,001$ \\
1. Tosse & 0,159 & Fraca & 0,490 \\
2. Crise de asma & 0,058 & Fraca & 0,860 \\
3. Chiado no peito & 0,014 & Fraca & 0,750 \\
4. Aperto no peito & 0,028 & Sofrível & 0,090 \\
5. Sintomas de respiração curta & 0,139 & Sofrível & 0,130 \\
6. Sentiu-se cansado & 0,133 & Sofrível & 0,450 \\
7. Acordou a noite por asma & 0,066 & Fraca & 0,010 \\
8. Sentiu-se sem respiração & 0,210 & Sofrível & $<0,001$ \\
9. Problemas para dormir & 0,288 & Sofrível & $<0,001$ \\
10. Dificuldades em respirar profundamente & 0,245 & Sofrível & $<0,001$ \\
Domínio das emoções & & & $<0,001$ \\
1. Sentiu-se frustrado & 0,371 & Sofrível & $<0,001$ \\
2. Sentiu-se preocupado & 0,361 & Sofrível & 0,920 \\
3. Sentiu-se zangado & 0,253 & Sofrível & Ruim \\
4. Emoções por sentir-se de fora & $-0,011$ & Fraca & 0,050 \\
5. Frustrado por não poder estar com os outros & 0,198 & Fraca & 0,600 \\
6. Desconfortável & 0,053 & Sofrível & $<0,001$ \\
7. Apavorado por crise de asma & 0,244 & Fraca & 0,920 \\
8. Sentiu-se irritado & 0,008 & & $<0,001$ \\
Domínio das atividades & & Sofrível & 0,240 \\
1. Incomodado por não poder estar com os outros & 0,235 & Fraca & \\
2. Incomodado ao realizar atividades na ultima semana & 0,102 & &
\end{tabular}

de fora" e "sentir-se irritado"; e no domínio atividades, a pior concordância foi observada no item "incomodado ao realizar atividades na última semana”. Os itens com melhor nível de concordância (sofrível) foram: sintomas ("problemas para dormir"), emoções ("sentiu-se frustrado" e "sentiu-se preocupado") e atividades ("incomodado por não poder estar com os outros").

Quanto à concordância entre adolescentes e seus pais, os resultados são apresentados na Tabela 4. No domínio sintomas, o item que teve pior concordância foi "sentiu-se sem respiração". Já para o item "problemas para dormir", a concordância foi perfeita entre pais e adolescentes. No domínio emoções, o item que teve pior concordância foi "sentir-se frustrado" e "irritado" e, no domínio atividades, o item com pior concordância foi "incomodado por não poder estar com os outros".

\section{Discussão}

A avaliação da QV de crianças e de adolescentes asmáticos e seus pais não teve uma concordância satisfatória na realidade pesquisada. Especula-se que este resultado possa ter sido influenciado pela dificuldade na compreensão de termos utilizados no questionário tanto pelas crianças e adolescentes, quanto por seus pais. A melhor concordância observada entre as respostas de adolescentes e pais deve ter ocorrido pela melhor capacidade de compreensão dos termos do questionário pelos adolescentes. Embora se espere que o adolescente tenha maior capacidade de verbalizar os seus sintomas e emoções, estudos apontam que a concordância com as respostas dos pais é prejudicada, já que os pais tendem a subestimar o impacto da doença sobre a vida do adolescente ${ }^{(12-14)}$. 
Tabela 4 - Concordância na aplicação do questionário PediatricAsthma Quality of Life Questionnaire Adaptado entre as respostas obtidas de adolescentes e seus pais

\begin{tabular}{lccc}
\hline & Coeficiente Kappa & Concordância & Valor $\boldsymbol{p}$ \\
\hline Domínio dos sintomas & & & \\
1. Tosse & 0,168 & Fraca & 0,050 \\
2. Crise de asma & 0,322 & Sofrível & $<0,001$ \\
3. Chiado no peito & 0,235 & Sofrível & 0,010 \\
4. Aperto no peito & 0,300 & Sofrível & $<0,001$ \\
5. Sintomas de respiração curta & 0,152 & Fraca & 0,100 \\
6. Sentiu-se cansado & 0,354 & Sofrível & $<0,001$ \\
7. Acordou a noite por asma & 0,170 & Fraca & 0,070 \\
8. Sentiu-se sem respiração & 0,032 & Fraca & 0,750 \\
9. Problemas para dormir & - & Perfeita & 0,530 \\
10. Dificuldades em respirar profundamente & 0,097 & Fraca & 0,340 \\
Domínio das emoções & & & 0,120 \\
1. Sentiu-se frustrado & 0,156 & Fraca & $<0,001$ \\
2. Sentiu-se preocupado & 0,397 & Sofrível & $<0,001$ \\
3. Sentiu-se zangado & 0,361 & Sofrível & 0,050 \\
4. Emoções por sentir-se de fora & 0,212 & Sofrível & 0,010 \\
5. Frustrado por não poder estar com os outros & 0,268 & Sofrível & $<0,001$ \\
6. Desconfortável & 0,425 & Regular & $0,010^{*}$ \\
7. Apavorado por crise de asma & 0,253 & Sofrível & 0,120 \\
8. Sentiu-se irritado & 0,156 & Fraca & \\
Domínio das atividades & & Ruim & 0,620 \\
1. Incomodado por não poder estar com os outros & $-0,051$ & Sofrível & $0,030^{*}$ \\
2. Incomodado ao realizar atividades na ultima semana & 0,231 & & \\
\hline
\end{tabular}

*Devido à impossibilidade de calcular o coeficiente Kappa (matriz de dados não simétrica), foi calculado o coeficiente de correlação de Spearman

A avaliação da QV é reconhecida por profissionais de saúde como um "elo" que tenta preencher a lacuna existente entre o diagnóstico e o manejo do tratamento clínico. O cuidado em saúde na asma brônquica, em crianças e adolescentes, não deve focar somente a sobrevida do paciente, mas a qualidade da extensão da vida, abrangendo aspectos importantes e fundamentais envolvidos na dinâmica familiar e percepção das experiências vivenciadas pelas crianças e seus pais ${ }^{(2,5,6)}$. Tais avaliações são feitas por instrumentos genéricos ou específicos. O instrumento utilizado para analisar a QV - o PAQLQ-A - é específico e foi escolhido porque objetiva compreender aspectos fundamentais para a adequada avaliação da QV. Associa-se ao fato de ter sido validado e adaptado à língua portuguesa, sendo considerado de boa aplicabilidade, podendo ser respondido pelo próprio paciente ou aplicado por entrevistador treinado ${ }^{(5)}$. No entanto, no presente estudo, o instrumento apresentou alguns problemas quanto à compreensão por parte de crianças, adolescentes e pais, possivelmente por questões culturais relacionadas ao entendimento da linguagem e alfabetização das crianças.

O estudo que apresentou a adaptação e a validação do questionário no Brasil apontou dificuldades na aplicação do instrumento para as idades de sete a oito anos e para algumas crianças com baixo nível socioeconômico, evidenciando as dificuldades por parte das crianças em entender alguns significados de palavras como "frustrado", "moderadamente" ou "desconfortável"(9). No nosso estudo, obtivemos resultados semelhantes à pesquisa de validação do instrumento no Brasil no que se refere a palavras e termos de difícil compreensão, como o termo "sentir-se de fora". Isto talvez tenha sido um dos motivos de a concordância ter sido sofrível entre os adolescentes e seus pais e ruim entre 
as crianças e seus pais, pois, principalmente neste item, a amostra foi reduzida.

Com relação à concordância das respostas das crianças e seus pais, percebe-se que os pais não mostram boa percepção do impacto da doença na vida de seus filhos. Talvez porque sua percepção se respalde na observação das manifestações clínicas da doença como tosse, dispneia, levantar noturno, uso das medicações broncodilatadoras, número de vezes que buscou atendimento de emergência no último mês, entre outros. A implicação deste achado é que, em crianças de sete a 12 anos, os clínicos não podem confiar totalmente no relato dos pais para o controle da QV das crianças. Sempre que as crianças fornecerem informações válidas e confiáveis sobre suas experiências da doença, estas devem ser consideradas. A impressão dos pais deve ser utilizada como informação complementar $^{(6,15)}$.

Em relação aos adolescentes e seus pais, os resultados mostram que os pais conseguem obter informações com o relato de experiências vividas no dia a dia de seus filhos, tendo em vista que as crianças possuem moderado entendimento sobre a doença. Os clínicos devem geralmente confiar no relato dos adolescentes, pois seus pais têm uma tendência a informar apenas o que observam e não o que os adolescentes realmente vivenciam $^{(13,14)}$.

Existem diferenças durante a aplicação do questionário a crianças e adolescentes quanto à compreensão do "conceito" do que é concreto e do que é subjetivo; ou seja, as faixas etárias menores podem fornecer relatos empiricamente confiáveis sobre o conceito de saúde, tais como dor e uso de medicações, mas não compreendem o conceito associado ao impacto emocional da doença, o que fica reservado, para as faixas etárias maiores e adolescentes ${ }^{(9,16,17)}$. O nível de compreensão da linguagem escrita e leitura também podem colocar um limite sobre a idade apropriada de aplicação de um questionário ${ }^{(9,16,17)}$. Ao avaliar a QV das crianças, é importante lembrar que as crianças estão envoltas em múltiplos contextos sociais, incluindo o relacionamento com seus pais e o funcionamento familiar, o seu grupo etário, a escola e a comunidade. Cada um destes contextos contribui para o impacto da doença e seu tratamento, sendo estes qualitativamente diferentes para crianças e adultos.

Para implementar as avaliações da QV relacionadas à saúde em pediatria, os pesquisadores precisam avaliar se a idade é apropriada para o instrumento, observando as instruções, o vocabulário, a estrutura da sentença, o conteúdo, as opções de resposta, os fatores contextuais, o desenvolvimento cognitivo e as diferenças culturais ${ }^{(8,18)}$. Outro ponto importante é que devido à maior variabilidade de respostas esperadas em crianças de faixas etárias menores, é necessária uma amostra maior para se determinar o efeito do tratamento na QV.

Desse modo, o determinante da avaliação da QV em crianças e adolescentes é complexo e esforços devem ser direcionados para avaliações tradicionais - clínicas e laboratoriais - bem como para avaliações de QV. Sempre que a criança for capaz de fornecer informações válidas e confiáveis, seu relato é a estratégia ideal, pois é consistente com a definição da $\mathrm{QV}$ relacionada à saúde que enfatiza a perspectiva subjetiva do paciente ${ }^{(9,11,13,19)}$. Alguns pesquisadores sugerem obter informações das crianças e de seus pais. Esta perspectiva pode fornecer o impacto da doença e do tratamento e sua influência na vida das crianças e adolescentes e seus familiares ${ }^{(8,20,21)}$.Estudos têm examinado esta questão ao avaliar o grau de concordância entre o relato dos pais e crianças. A suposição é de que um alto nível de concordância indicaria que tanto a criança/adolescente quanto os pais poderiam responder questionários para avaliação da QV de crianças e adolescentes $^{(4,16,17,22,23)}$. Entretanto, esta concordância satisfatória não foi encontrada no presente estudo, indicando que, principalmente no grupo etário das crianças, a percepção dos pais sobre a QV da criança asmática difere muito daquela percebida pela criança. Com relação aos adolescentes, constatou-se uma melhor concordância, embora perfeita em apenas um item, relacionado à dificuldade para dormir. Entretanto, no domínio atividades do adolescente foi obtida a pior concordância entre as respostas deles e de seus pais.

No caso de discordância, os pesquisadores e profissionais de saúde necessitam determinar qual o relato é mais confiável ou apropriado para os estudos. O grau de concordância pode depender de vários fatores, ente eles, os domínios do questionário aplicado, a idade da criança e do adolescente e o entendimento da percepção do processo de saúde e doença da criança pelos pais ou cuidadores próximos. Por exemplo, a concordância tem se mostrado mais elevada para os domínios físicos do que para os emocionais. Com relação à idade, a faixa etária mais elevada se associa à maior concordância ${ }^{(16,24)}$, aspecto corroborado pelo presente estudo.

Outra questão relevante, e não bem definida, é se os pais são os respondentes adultos próximos mais apropriados. Algumas crianças podem passar mais tempo com o professor, babá ou outro membro da família do que com os pais, e este cuidador pode ter opinião mais acurada do ponto de vista fisiológico e social da criança ${ }^{(8,21,25)}$. No Brasil, faltam dados quanto à confiabilidade das informações fornecidas pelo cuidador. 
A QV é considerada, portanto, uma medida de desfecho clínico, que prioriza a avaliação subjetiva do próprio paciente quanto aos efeitos que uma doença ou um tratamento exercem sobre sua vida diária e seu nível de satisfação e bem-estar ${ }^{(5,26)}$. O estudo aponta aspectos a serem considerados na aplicação de instrumentos elaborados e validados para avaliação da QV nas faixas etárias que compreendem a infância e adolescência. O primeiro aspecto se deve aos instrumentos genéricos e específicos utilizados, os quais são derivados de outros países e validados nacionalmente. É preciso levar em conta as imensas diferenças regionais no Brasil, tornando algumas questões confusas e sem entendimento em diferentes localidades. Outro aspecto a ser considerado é o desenvolvimento cognitivo de crianças e adolescentes, que necessita ser respeitado ao se avaliar a QV de crianças e adolescentes asmáticos, tendo em

\section{Referências bibliográficas}

1. Autoria não referida. IV Brazilian Guidelines for the management of asthma. J Bras Pneumol 2006;32 (Suppl 7):S447-74.

2. Carr AJ, Gibson B, Robinson PG. Measuring quality of life: is quality of life determined by expectations or experience? BMJ 2001;322:1240-3.

3. Juniper EF. How important is quality of life in pediatric asthma? Pediatr Pulmonol 1997;15 (Suppl):17-21.

4. Guyatt GH, Juniper EF, Griffith LE, Feeny DH, Ferrie PJ. Children and adult perceptions of childhood asthma. Pediatrics 1997;99:165-8.

5. Willke RJ, Burke LB, Erickson P. Measuring treatment impact: a review of patient-reported outcomes and other efficacy endpoints in approved product labels. Control Clin Trials 2004;25:535-52.

6. Segre M, Ferraz FC. The concept of health. Rev Saude Publica 1997;31:538-42.

7. Juniper EF, Guyatt GH, Epstein RS, Ferrie PJ, Jaeschke R, Hiller TK. Evaluation of impairment of health related quality of life in asthma: development of questionnaire for use in clinical trials. Thorax 1992;47:76-83.

8. Juniper EF, Guyatt GH, Feeny DH, Ferrie PJ, Griffith LE, Townsend M. Measuring quality of life in the parents of children with asthma. Qual Life Res 1996;5:27-34.

9. La Scala CS, Naspitz CK, Solé D. Adaptation and validation of the Pediatric Asthma Quality of Life Questionnaire (PAQLQ) in Brazilian asthmatic children and adolescents. J Pediatr (Rio J) 2005:81:54-60.

10. Brasil - Secretaria de Atenção à Saúde - Departamento de Ações Programáticas Estratégicas. Marco teórico e referencial: saúde sexual e saúde reprodutiva de adolescentes e jovens. Brasília: Ministério da Saúde; 2006.

11. Andrade AL, Zicker F. Estudos de prevalência. In: Organização Panamericana da Saúde. Fundação Nacional de Saúde. Métodos de investigação epidemiológica em doenças transmissíveis: manual do instrutor. Brasília: Funasa; 1997. p. 33-42.

12. Cassol VE, Solé D, Menna-Barreto SS, Teche SP, Rizzato TM, Maldonado $\mathrm{M}$ et al. Prevalence of asthma among adolescents in the city of Santa Maria, in the state of Rio Grande do Sul, Brazil. International Study of Asthma and Allergies in Childhood (ISAAC) Project. J Bras Pneumol 2005;31:191-6.

13. Wright JG, Young NL. A comparison of different indices of responsiveness. J Clin Epidemiol 1997;50:239-46. vista que o autorrelato dos pacientes precisa ser analisado, complementando-se com informações advindas dos pais.

O PAQLQ-A não foi bem compreendido na realidade estudada e a concordância entre as respostas dos pais e das crianças e adolescentes não foi satisfatória, sendo de pior qualidade para o grupo etário de crianças. Para minimizar os problemas relacionados à aplicação de instrumentos internacionais validados, recomenda-se a elaboração de instrumentos adequados às especificidades regionais ou ainda a revalidação dos internacionais de forma que atendam às necessidades regionais. Adicionalmente, indica-se a elaboração de estratégias comunicacionais entre os profissionais de saúde, crianças e pais, com o objetivo de melhor captar o impacto produzido pela asma nas várias dimensões de vida das crianças, adolescentes e famílias que convivem com esta doença.

14. Katz JN, Larson MG, Phillips CB, Fossel AH, Liang MH. Comparative measurement sensitivity of short and longer health status instruments. Med Care 1992;30:917-25.

15. Fernandes AL, Oliveira MA. Avaliação da qualidade de vida na asma. J Pneumol 1997;23:148-52.

16. Theunissen NC, Volges TG, Koopman HM, Verrips GH, Zwinderman KA, Verloove-Vanhorick SP et al. The proxy problem: child report versus parent report in health-related quality of life research. Qual Life Res 1998;7:387-97.

17. Rebok G, Riley A, Forrest C, Starfield B, Green B, Robertson J et al. Elementary school-aged children's reports of their health: a cognitive interviewing study. Qual Life Res 2001;10:59-70.

18. Reichenberg K, Broberg AG. Quality of life in childhood asthma: use of the Paediatric Asthma Quality of Life Questionnaire in a Swedish sample of children 7 to 9 years old. Acta Paediatr 2000;89:989-95.

19. Kirkwood BR. Essentials of medical statistics. Oxford: Blackwell Science; 1988.

20. Burbach DJ, Peterson L. Children's concepts of physical illness: a review and critique of the cognitive-developmental literature. Health Psychol 1986;5:307-25.

21. Eiser C, Morse R. Can parents rate their child's health-related quality of life? Results of a systematic review. Qual Life Res 2001;10:347-57.

22. Clarke SA, Eiser $C$. The measurement of health-related quality of life (QOL) in paediatric clinical trials: a systematic review. Health Qual Life Outcomes 2004;2:66.

23. Annett RD. Assessment of health status and quality of life outcomes for children with asthma. J Allergy Clin Immunol 2001;107 (Suppl 5):S473-81.

24. April KT, Feldman DE, Platt RW, Duffy CM. Comparison between children with Juvenile Idiopathic Arthritis (JIA) and their parents concerning perceived Quality of Life. Qual Life Res 2006;15:655-61.

25. Osman LM, Baxter-Jones AD, Helms PJ; EASE Study Group. Parents' quality of life and respiratory symptoms in young children with mild wheeze. Eur Respir J 2001;17:254-8.

26. Christakis DA, Johnston BD, Connell FA. Methodologic issues in pediatric outcomes research. Ambul Pediatr 2001;1:59-62. 